\title{
Supervision and professional development in social work practice
}

\author{
Amanda Nickson, Margaret-Anne Carter and Abraham P. Francis \\ SAGE, 2020 \\ ISBN: 978-93-532-8663-7, pp.275, hardback, NZD140.00
}

$\mathrm{T}$ he space for supervision within the current context of neoliberalism and managerial practises compromise professional development and critical reflection. This book provides a solid contribution towards developing frameworks and ideas for students, practitioners, and supervisors related to supervision. Equally, diverse perspectives and research related to supervision are captured and generate an interest for more experienced practitioners, supervisors and academics. As the book's title suggests, there is a specific focus on social work, supervision and professional development within an Australian-Pacific context. However, connections are made throughout to a range of helping professions and the transferability of ideas, skills and approaches in supervision.

The book is in hardcover and well-presented, although the cost for this version is expensive. The content of the book presents as easy to read, combining theory to diverse contexts and enabling the reader to consider its relevance to their own practice. The book is unique in that it captures "voices from the field" related to supervision and associated topics. These voices are from students, practitioners, supervisors and academics who provide rich and diverse perspectives from their own experiences and offer their practice wisdom. At the conclusion of the chapters, exercises and reflective questions are provided to facilitate helpful learning and reflection for the reader. Noteworthy in this book are key links to websites, further readings at the end of each chapter, appendices, an index and helpful tables and diagrams for the reader's future reference.
The foreword is written by Lambert Engelbrecht, a key contributor in the area of supervision, where he summarises the significance of this book. The author's preface also signposts the outline and intention of the book's chapters. Each chapter has a specific focus and readers are guided towards specific interest areas in supervision without the need to read the book sequentially.

The first five chapters provide the significance of supervision, its definition, key debates, an important theoretical guide to supervision along with specific modes, models and approaches to supervision. Students, practitioners (particularly social workers) and new supervisors will find these discussions related to Australian, British and American Codes of Ethics for social workers important in setting the professional context for supervision. Well known Aotearoa New Zealand author, Kieran O'Donoghue's voice from the field is very engaging in Chapter Three where he discusses the two influences on supervision-professionalisation of social work and managerialism. Peer group supervision is explored in Chapter Four as a particular mode for supervision. Models and approaches such as the four-layered practice model for reflective supervision, developmental models, strengths-based approaches and the seven-eyed model are highlighted, although scattered and appear haphazardly through the initial chapters, particularly Chapter Four and Chapter Five.

Chapters Six to Eight explore the essential nature of professional growth and the relevance to supervision; culture 
and diversity; and the challenges and opportunities for supervision within a range of practice contexts. Chapter Seven, which explores diversity in supervision is compelling. The RESPECTFUL framework referred to offers reflection around multicultural meaning and dimensions together with thoughtprovoking voices from the field: working alongside Torres Strait Islanders; the perils of operating with a white lens in supervision; and LGBTIQ.

The book ends with chapters discussing the ever-changing online landscape of supervision (particularly relevant to Covid-19) and the need to harness compassion, resilience and make the most of reflective supervision. Key up-todate research is included. Chapter Ten's "compassionate supervision" is thought provoking as the reader is led on a journey exploring self-compassion and its relevance within an organisational framework and in supervision. Petrina May Barson's voice on sustaining compassion is heart-warming - we are reminded that sustaining ourselves needs to include loving kindness, a common sense of humanity and mindfulness. The risks and protective factors of compassion fatigue and compassion satisfaction are outlined in Chapter Eleven with some useful exercises. Some important voices include those of supervisors and students around the significance of coping, self-care and reflection. Specifically, Dana Esperanza's explores meaning in professional work and whether it brings joy or annoys. The final chapter repeats core messages from throughout the book: building effective relationships, setting goals and getting the most out of supervision.

Literature about supervision and its significance to professional practice is gathering momentum-reflecting the growing understanding and acknowledgment of different practice contexts. The book provides an important overview of supervision and aids students, social work practitioners and supervisors compile a deeper appreciation of the scope asupporting communities. 